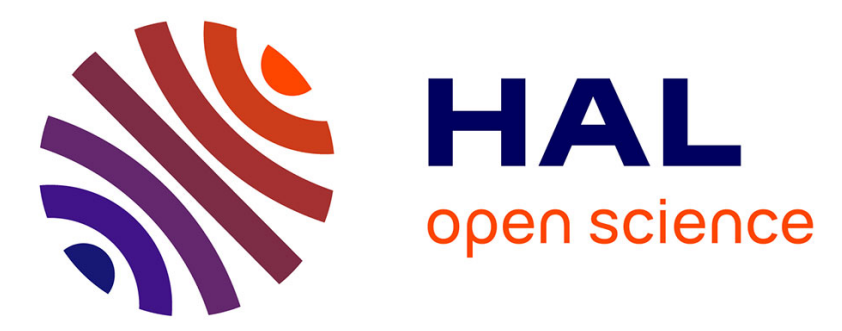

\title{
Small-angle X-ray scattering intensity of multiscale models of spheres
}

Loïc Sorbier, Maxime Moreaud, Severine Humbert

\section{To cite this version:}

Loïc Sorbier, Maxime Moreaud, Severine Humbert. Small-angle X-ray scattering intensity of multiscale models of spheres. Journal of Applied Crystallography, 2019, 52 (6), pp.1348-1357. 10.1107/S1600576719013839 . hal-02417477

\section{HAL Id: hal-02417477 \\ https://hal-ifp.archives-ouvertes.fr/hal-02417477}

Submitted on 18 Dec 2019

HAL is a multi-disciplinary open access archive for the deposit and dissemination of scientific research documents, whether they are published or not. The documents may come from teaching and research institutions in France or abroad, or from public or private research centers.
L'archive ouverte pluridisciplinaire HAL, est destinée au dépôt et à la diffusion de documents scientifiques de niveau recherche, publiés ou non, émanant des établissements d'enseignement et de recherche français ou étrangers, des laboratoires publics ou privés. 


\title{
Small Angle X-ray Scattering intensity of multiscale models of spheres
}

\author{
LoḮ Sorbier, ${ }^{a *}$ Maxime Moreaud $^{a, b}$ And Séverine Humbert ${ }^{a}$ \\ ${ }^{a}$ IFP Energies nouvelles, Rond-point de l'échangeur de Solaize, BP 3, 69360 Solaize, \\ France, and ${ }^{b}$ MINES ParisTech, PSL-Research University, CMM, 35 rue Saint \\ Honoré, 77305 Fontainebleau, France. E-mail: loic.sorbier@ifpen.fr
}

\begin{abstract}
The different approaches found in literature to compute small angle X-ray scattering intensities of stochastic Boolean models from their analytical formulations or their numerical realizations are reviewed. The advantages and drawbacks of the methods for the interpretation of small angle X-ray scattering curves are investigated. Examples of multiscale models built from union and intersection of Boolean models of spheres and from Gamma or log-normal radius distribution are given. The scattered intensity computed from projections of realizations of such models are compared with the intensity computed from their analytical covariance. It appears that computation from projection induces strong finite size effect with a relative variance constant and equal to 0.5. Comparison of scattering intensities of an intersection of Boolean model and the corresponding Cox model shows only subtle differences.
\end{abstract}

PREPRINT: Journal of Applied Crystallography A Journal of the International Union of Crystallography 


\section{Introduction}

Heterogeneous catalysts are of primary importance in the production of chemicals (Dingerdissen et al., 2008). These catalysts are often formed by a porous support covered by active phases constituted of particles of varying shapes and sizes ranging from the nanometer scale to the micrometer scale (Weckhuysen, 2009). Many supports of heterogeneous catalysts may be viewed as a collection of grains randomly stacked, the volume between the grains giving rise to the porosity. For example, alumina supports may be modeled as nanometric platelets that are aggregated at a larger scale (Wang et al., 2015). Likewise, the active phase could also be seen as an assembly of particles. For example, Fischer-Tropsch catalysts are composed of cobalt nanometric particles that are themselves grouped into aggregates on a silica-alumina support (Humbert et al., 2018). The two-scales organization of the active phase of catalysts can be related to their performance (activity, selectivity) (Munnik et al., 2014). The particles of support or active phases are often polydisperse, with a normal or log-normal distribution law depending if they are formed by coalescence or ripening (Granqvist \& Buhrman, 1976; Datye et al., 2006). Morphological details of catalysts may be observed at the nanometer scale with Transmission Electron Microscopy (TEM) (Thomas \& Terasaki, 2002) or Electron Tomography (ET) (Friedrich et al., 2009) and at the micrometer scale with Scanning Electron Microscopy (SEM) (Lomić et al., 2004) coupled or not with Focused Ion Beam (FIB) (Witte et al., 2013). However, the extraction of a particle size distribution is tedious and these techniques are often limited by their lack of representativeness.

Small-Angle X-ray Scattering (SAXS) probes the fluctuation of electron density in a material (Li et al., 2016) at a scale ranging between one and few hundreds of nanometer and within a volume in the order of slightly less than the $\mathrm{mm}^{3}$. Therefore, it is an interesting technique for the characterization of catalytic systems, if it can handle 
polydisperse spherical and/or platelet-like particles that are more or less aggregated within aggregates more or less dense. This is a crucial point that differs from other systems usually analyzed by SAXS.

Indeed, SAXS has been widely employed for the characterization of colloidal suspension and materials like cement, metallic nanoparticles, oil, polymers, pharmaceuticals, food or proteins. SAXS relates the intensity $I$ scattered at a wave vector $\boldsymbol{q}$ with the Debye correlation function or normalized covariance of the sample $\gamma(\boldsymbol{r})$. Conventional data processing involves a split of the scattered intensity into form and structure factors, very suitable for dilute suspensions, form factor being restricted to simple morphologies and structure factor generally to hard-core repulsion. However, for microstructures such as porous media or active phases encountered in heterogeneous catalysis, conventional data processing is not relevant. For example, the multi-scale models proposed by Beaucage (1995) are not suited for non-fractal morphologies encountered in heterogeneous catalysis. An approach implemented in the program MIXTURE (Konarev et al., 2003) allows to fit the experimental scattering curve by modeling the multicomponent system with different form factor and taking into account interparticle interactions. These interactions are described by structure factor calculated within Percus-Yevick approximation for hard-sphere or sticky hardsphere potential. However the modeling of aggregated particles is not obvious and the consideration of platelet-like objects, or size distribution such as gamma or log-normal distribution functions, are not available. Besides, an other approach is proposed by Bressler et al. (2015) with the McSAS software and gives a distribution of an arbitrary shape from a single form factor.

Exploitation of the SAXS intensity can also be performed thanks to Boolean models, either by direct calculation of $I(\boldsymbol{q})$ from a known covariance or by inverting the relation to extract $\gamma(\boldsymbol{r})$. The first approach has been applied on one-scale Boolean 
model of spheres (Gille, 2011), union and intersection of Boolean models, dead leaves model, clipped Gaussian field and Gaussian field intersection model (Gommes, 2018). The second approach has been applied for one-scale Boolean model of sphere with monodisperse or exponential size distribution, Poisson polyhedra, intersection of two Poisson polyhedra models and the intersection of a Poisson polyhedron and the complementary of Poisson polyhedron (Sonntag et al., 1981). Both approaches are computationally efficient as they only require a single one-dimensional Fourier transform and a numerical differentiation to obtain the SAXS intensity. They are however limited to models with an analytical covariance. These works are restricted to models with known analytical covariance and very simple particle size distribution (Sonntag et al., 1981; Gille, 2011) or arbitrary but discrete distribution (Gommes, 2018).

Whatever the approach, it is worth noticing that a matching correlation function does not completely defines a microstructure (Gommes et al., 2012; Gommes, 2018). One of the advantages of using morphological models such as Boolean models for materials modeling is that some of their parameters can be constrained either by knowledge of the synthesis process or from complementary characterization techniques (microscopy, nitrogen adsorption or mercury porosimetry). This can dramatically reduce the microstructures matching with a specified covariance. This is the strength of this kind of approach compared to the conventional data processing. Moreover Boolean models can handle any particle shape and may be combined to model multi-scale microstructures in an easy way.

The aim of this paper is thus to propose to interpret SAXS intensity with more elaborate intersection and/or union of Boolean models and through multi-scale Boolean models with Cox point processes of arbitrary complexity. The first section recalls the basic equations for Boolean models. Next section reviews the different approach to compute SAXS intensity from a known microstructure. We show in third section

IUCr macros version 2.1.11: 2019/01/14 
results for one-scale Boolean models of spheres, Boolean models of spheres with a distribution in radius, model of aggregated and isolated particles and differences between intersection of Boolean models and their corresponding Cox model. The fourth section is devoted to the discussion of Representative Volume Element (RVE) and the numerical cost of the proposed approaches.

\section{Boolean models}

\subsection{One-scale Boolean models}

The principle of a Boolean scheme is to generate random Poisson points with a $\theta$ density and implanting on each point a primary grain that can be stochastic (Matheron, 1967; Matheron, 1975). Notice that the grains can overlap each other. In this manuscript we will restrict to the case of a biphasic material where solid fraction has a finite $\rho>0$ electronic density and a void fraction with a $\rho=0$ electronic density,

otherwise said, a porous material. The approach can however be easily extrapolated to materials with more than two phases.

The volume fraction $p$ of a Boolean set is related with the intensity of the process $\theta$ by:

$$
p=1-e^{-\theta E\left[V_{\mathrm{G}}\right]}
$$

where $E\left[V_{\mathrm{G}}\right]$ is the expectation of the volume of the primary grains. The porosity of the model $\varepsilon=1-p$ is trivially related to the volume fraction $p$.

The two-points correlation function of the set (of the solid phase) $C_{11}(h)$ is the probability that two points that are separated by a distance $h$ belong to the set. It reads:

$$
C_{11}(h)=\varepsilon^{2} e^{\theta K(h)}+1-2 \varepsilon
$$

where $K(h)$ is the geometrical covariogram of the primary grain defined as the expectation of the volume of the intersection of the grain with its translated by vectors of IUCr macros version 2.1.11: 2019/01/14 
module $h$. For spherical grains of constant radius $R$ :

$$
K_{\mathrm{S}}(R, h)=\frac{4 \pi}{3}\left(R^{3}-\frac{3}{4} h R^{2}+\frac{h^{3}}{16}\right) \Theta(2 R-h)
$$

with $\Theta$ the Heaviside's step function.

The two-points correlation function of the complementary of the set (of the pores) $C_{00}(h)$ is the probability that two points that are separated by a distance $h$ belong to the complementary of the set. It is related to $C_{11}(h)$ and to the normalized covariance $\gamma(h)$ by:

$$
C_{00}(h)-\varepsilon^{2}=C_{11}(h)-p^{2}=p \varepsilon \gamma(h)
$$

Thus, the normalized covariance of a Boolean model reads:

$$
\gamma(h)=\frac{1-p}{p}\left(e^{\theta K(h)}-1\right)
$$

\subsection{Union and intersection of models}

Union and intersection of Boolean models allow to produce more elaborate models while keeping tractable analytical formula.

Union allows to produce models with a distribution in size or in shape of the primary grains. As a point belongs to the complementary of the union of $n$ models, if and only if it belongs to the complementary of each model, we have:

$$
\begin{gathered}
\varepsilon=\prod_{i=1}^{n} \varepsilon^{(i)} \\
C_{00}(h)=\prod_{i=1}^{n} C_{00}^{(i)}(h)
\end{gathered}
$$

One consequence of these relations is that a model with a continuous grain size distribution behaves like a one-scale model with a modified geometrical covariogram. For a Boolean model of spheres which radius follow a distribution law $P(R)$, the geometrical covariogram reads:

$$
K(h)=\int_{0}^{\infty} P(R) K_{\mathrm{S}}(R, h) \mathrm{d} R
$$


Let $S_{P}^{n}(x)$ defined by:

$$
S_{P}^{n}(x)=\int_{x}^{\infty} R^{n} P(R) \mathrm{d} R
$$

With this definition, $S_{P}^{n}(0)$ is the $n$th uncentered moment of $P$. We have:

$$
E\left(V_{\mathrm{G}}\right)=\frac{4 \pi}{3} S_{P}^{3}(0)
$$

The volume-averaged radius $R_{\mathrm{V}}$ writes:

$$
R_{\mathrm{V}}=\frac{S_{P}^{4}(0)}{S_{P}^{3}(0)}
$$

And the covariogram reads:

$$
K(h)=\frac{4 \pi}{3}\left[S_{P}^{3}\left(\frac{h}{2}\right)-\frac{3}{4} h S_{P}^{2}\left(\frac{h}{2}\right)+\frac{1}{16} h^{3} S_{P}^{0}\left(\frac{h}{2}\right)\right]
$$

If the analytical formulations of $S_{P}^{n}(x)$ are known, the covariance may be easily calculated from equations 12 and 5. The expressions of the covariogram for Boolean model of spheres are given explicitly in supplementary materials for the log-normal, the Gamma and the exponential distributions of radius.

Intersection allows the building of models of aggregated primary grains. As a point belongs to an intersection of $n$ models, if and only if it belongs to each model, we have:

$$
\begin{gathered}
p=\prod_{i=1}^{n} p^{(i)} \\
C_{11}(h)=\prod_{i=1}^{n} C_{11}^{(i)}(h)
\end{gathered}
$$

Union and intersection of models may be combined to obtain even more complex microstructures. For example, the intersection of a large scale model with a smaller scale model generates aggregated particles. The union of this model with the intersection of the large scale model with another small scale model gives a model with isolated and aggregated particles. This kind of models have been used to model ASAXS intensity of cobalt based catalyst trough analytical models neglecting structure factors (Humbert et al., 2018). 


\subsection{Cox models}

Multi-scales Boolean models may be obtained by intersection of Boolean models. Unfortunately, this strategy leads to unrealistic morphologies where some primary grains are cut. Another slightly different way of approaching the problem allows to obtain more realistic microstructures. The shape the primary grains can be kept if the Poisson seeds associated to the grain are implanted in the large-scale Boolean model first and the primary grains implanted on the seeds afterwards. This type of model uses Cox point processes (Jeulin, 1996; Jeulin, 2012). To make an intersection of two models, only the grains of the first model whose seeds fall inside the second model are totally preserved. More details can be found in (Moreaud, 2006). Although analytical covariance of such composition of models are not known, we will see later that this approach is of interest if we take the problem from a numerical point of view.

\section{Methods to compute SAXS intensity}

Methods to compute SAXS intensity from a microstructure depends on the complexity of the microstructure and relies on Fourier transforms:

- One dimensional Fourier transform if the covariance is known (analytical covariance);

- One or two-dimensional Fourier transform after linear projection of an arbitrary microstructure;

- Three-dimensional Fourier transform for arbitrary microstructure by numerical evaluation of the covariance;

For the remainder of the document, microstructures are considered as defined by a binary function $\chi_{b}: \mathbb{R}^{3} \rightarrow\{0 ; 1\}$ with points representing matter defined by the set $\mathrm{X}=\left\{\boldsymbol{x} \in \mathbb{R}^{3} \mid \chi_{b}(\boldsymbol{x})=1\right\} ; \mathrm{X}$ is a bounded set. Let $\partial \gamma$ the convex hull of $\mathrm{X}$ with $\gamma$ a bounded set of $\mathbb{R}^{3}$, defined as the smallest convex set such as $\mathrm{X} \subset \gamma$. Finally 
let $\chi$ be a 3D binary image representing the microstructure, defined by $\chi_{b}$ and $\gamma$ by $\chi: \gamma \rightarrow\{0 ; 1\} ; \boldsymbol{x} \mapsto \chi_{b}(\boldsymbol{x})$, otherwise said $\chi$ is a restriction of $\chi_{b}$ to $\gamma$.

\subsection{Analytical covariance}

Defining the Fourier transform $\mathcal{F}(g)$ of $g$ by:

$$
\mathcal{F}(g)(q)=\int_{-\infty}^{+\infty} g(r) e^{-i q r} \mathrm{~d} r
$$

the SAXS intensity reduces to (Levitz \& Tchoubar, 1992):

$$
I(q)=I_{\mathrm{e}}(q) V p(1-p) \rho^{2} \frac{-2 \pi}{q} \frac{\partial}{\partial q}[\Re[\mathcal{F}(\gamma)(q)]]
$$

where $q$ is the wave vector of diffusion, $I_{\mathrm{e}}(q)$ the intensity scattered by one electron, $V$ the volume irradiated by the incident X-ray beam and $\Re(z)$ the real part of $z$. Equation 16 may be helpful to compute SAXS intensity from a known covariance. It implies a one-dimensional Fourier transform and a numerical differentiation. It can be evaluated numerically by an efficient Fast Fourier Transform algorithm (FFT).

\subsection{Computation of the SAXS intensity from projection}

Brisard et al. (2012) invoking the Fourier slice theorem (Kak \& Slaney, 1988) indicate that the SAXS intensity is proportional to the square of the modulus of the Fourier transform of the linear projection of the microstructure. In the tomography literature, the linear projection of a function $f(\boldsymbol{r})$ is defined as the integral path of the function along a direction. For example the linear projection $\mathcal{P}_{z}[f]$ of $f(\boldsymbol{r})$ along the $z$ axis in Cartesian coordinates is defined by:

$$
\mathcal{P}_{z}[f](x, y)=\int_{-\infty}^{\infty} f(x, y, z) \mathrm{d} z
$$

Assuming a biphasic porous medium with indicator function $\chi(\boldsymbol{r})$ the SAXS intensity in the $q_{z}=0$ plane reads (see supplementary materials):

$$
\frac{I\left(q_{x}, q_{y}\right)}{I_{\mathrm{e}}\left(q_{x}, q_{y}\right)}=\rho^{2}\left|\mathcal{F}\left(\mathcal{P}_{z}[\chi](x, y)\right)\right|^{2}
$$

IUCr macros version 2.1.11: 2019/01/14 
For a statistically isotropic medium, the SAXS intensity may be evaluated in any plane as it depends only on the modulus $q$ of the scattering vector. Thus, the SAXS intensity of an isotropic medium is easily calculable from the projection of the microstructure along an arbitrary direction. The general methods to compute the SAXS intensity from projection are the following, depending on the anisotropy of the microstructure.

For statistically isotropic systems:

- Generate a periodic, digital realization of the microstructure on a finite 3D volume: $\chi(x, y, z)$;

- Compute the linear projection of the realization along an arbitrary axis, for example the $z$ axis using Equation 17 , obtaining the 2D image $\mathcal{P}_{z}[\chi](x, y)$;

- Compute the square modulus of the Fourier transform of this projection using a 2D FFT algorithm in order to obtain $I\left(q_{x}, q_{y}\right)$ (Equation 18;

- Compute $I(q)=\frac{1}{2}[I(q, 0)+I(0, q)]$

For anisotropic systems having the $y$ and $z$ equivalent directions:

- Generate a periodic, digital realization of the microstructure on a finite 3D volume: $\chi(x, y, z)$;

- Compute the linear projection of the realization along either $y$ or $z$ axis, for example the $z$ axis using Equation 17 , obtaining the 2D image $\mathcal{P}_{z}[\chi](x, y)$;

- Compute the square modulus of the Fourier transform of the projection using a 2D FFT algorithm to obtain $I\left(q_{x}, q_{y}\right)$ (Equation 18);

An only two dimensional Fourier transform is needed. Besides, any type of complex multi-scale microstructures can be used with this approach, such as previously presented combination of Boolean models and Cox point processes. At last, for isotropic systems, an improvement of the method could be not to reconstruct the complete microstructure but only a linear projection of it along an arbitrary direction.

IUCr macros version 2.1.11: 2019/01/14 


\subsection{Numerical evaluation of the covariance}

For periodic microstructures, the covariance may be evaluated by Fast Fourier Transform (FFT) (Koch et al., 2003; Schmidt-Rohr, 2007):

$$
C_{11}(\boldsymbol{h})=\mathcal{F}^{-1}\left(|\mathcal{F}(\chi(\boldsymbol{h}))|^{2}\right)
$$

Combining equations 16 and 19 , the scattered intensity reads:

$$
I(q)=I_{\mathrm{e}}(q) V \rho^{2} \frac{-2 \pi}{q} \frac{\partial}{\partial q}\left[|\mathcal{F}(\chi(\boldsymbol{h}))|^{2}\right]
$$

The SAXS intensity may be computed from a three-dimensional Fourier transform and a numerical differentiation. As this method implies a three-dimensional FFT, it requires long computation time and large memory requirement and was not explored in this work.

\subsection{Variance of computed SAXS intensity}

The software platform plug im! (Moreaud, 2018) has been used to produce binarized realizations of Boolean models of spheres with radius constant or distributed according to a Gamma law and union and/or intersection of them. The realizations are periodic within the cell defined as a cubic volume of side $N$. The computation of the projection and calculation of the SAXS intensity from it has been implemented as a module in this platform using Fast Fourier Transform. Typical running times (not specially optimized) for generating one realization on a $2.3 \mathrm{GHz}$ Intel Xeon ES-2650 workstation with 32 Go memory are given in Table 1. Projection of a realization last about 3 $\mathrm{s}$ and the computation of the SAXS intensity less than one second. Computation of SAXS intensity from covariance for Boolean model of spheres and their union and/or intersection has been implemented on another module for the platform using Fast Fourier Transform. Typical running time is less than one second for a $2^{18}$ wide simulation domain.

IUCr macros version 2.1.11: 2019/01/14 
Methods to compute SAXS intensity that relies on the generation of realizations of models (such as computation of SAXS diagram from projection) will introduce variability in their results. To quantify the uncertainty of the SAXS intensity computed from projection $I_{\text {proj }}(q)$, we define the relative variance $\mathcal{V}(q)$ by:

$$
\mathcal{V}(q)=\frac{\operatorname{Var}\left[I_{\text {proj }}(q)\right]}{E\left[I_{\text {proj }}(q)\right]^{2}}
$$

The variance $\mathcal{V}(q)$, will allow us to study the relative precision of an averaged intensity obtained from these $n$ realizations. Indeed, the relative uncertainty of the averaged intensity is expected to scale as $\sqrt{\mathcal{V}(q) / n}$.

\section{Results}

Calculated SAXS intensities will be reported plotting the intensities normalized by the intensity at $q=0$ versus the wave vector $q$ normalized by the inverse of the characteristic length scale $1 / R_{\mathrm{V}}$ with $R_{\mathrm{V}}$ the volume-averaged radius of the primary spheres. All length scales are in voxel units and $q$ scales in voxel $^{-1}$ units.

\subsection{Boolean model of spheres with constant radius}

Figure 1 shows two surface renderings of one realization of a Boolean model of spheres with constant radius $R=20$ for 0.2 and 0.8 volume fractions. Figure 2 shows the comparison of the SAXS intensity of a one-scale Boolean model of spheres of constant radius $R$ for volume fraction $p=0.8$ calculated from equations 16 and 18 , A single realization leads to oscillations of the intensity around the expected value. When $q \gtrsim 1$, intensity computed from projection starts to differ significantly from intensity computed from analytical covariance. Figure 3 shows the same comparison but from the mean SAXS intensity of 1000 independent realizations. A much better agreement is found between both methods, the oscillations for the method by projection are IUCr macros version 2.1.11: 2019/01/14 
much less pronounced than for a single realization. The $q^{-4}$ dependence at high $q$, characteristic of smooth isotropic surfaces is observed as expected. A departure from the expected intensity at high $q$ is observed. Figure 4 shows the variance of the bias. Variance is found to be constant for the whole $q$ range and close to 0.5 for any volume fraction $p$ and any size of simulation domain.

\subsection{Boolean model of spheres with radius following a Gamma distribution}

Figure 5 shows two surface renderings of one realization of a Boolean model of spheres with radius following a Gamma distribution with scale parameter $b=4$ and shape parameter $c=2$ for 0.2 and 0.8 volume fractions. $b$ is the mean radius (in number) and $c$ is an adimensional parameter controlling the distribution width. These parameters were chosen to obtain the same mean volume radius $R_{\mathrm{V}}=20$ than for the previous model with constant diameter. Figure 6 shows the comparison between the SAXS intensity calculated from analytical covariance (equation 16 ) and from projection (equation 18) of a single realization of a one-scale Boolean model of spheres following this Gamma distribution. The volume mean radius of the spheres is 20 pixels in a $1024^{3}$ voxels volume. Marked oscillations are noticeable. Figure 7 shows the same comparison but with the mean SAXS intensity of 1000 independent realizations of the model. The $q^{-4}$ dependence at high $q$, characteristic of smooth isotropic surfaces is well recovered and the spurious oscillations are less visible. As for the previous cases, intensity computed from projection differs significantly from intensity computed

from analytical covariance when $q \gtrsim 1$. At small $q$, the calculated intensity is slightly underestimated

IUCr macros version 2.1.11: 2019/01/14 


\subsection{Boolean model of spheres with large distribution in radius}

Log-normal distribution allows to generate Boolean model of spheres with a very wide radius distribution. The relative standard deviation $(R S D)$ of the volume-averaged radius of spheres for a log-normal distribution of parameters $\mu$ and $\sigma$ is given by:

$$
R S D=\sqrt{e^{\sigma^{2}}-1}
$$

Figure 8 shows the SAXS intensity of Boolean models of spheres with a fixed volumeaveraged radius $R_{\mathrm{V}}$ and variable $R S D$. Large $R S D$ results in an intensity with a pseudo-fractal behavior on a large range of $q$. For such models, the scattered intensity scales as a power law $q^{-\alpha}$ in the range $q R_{\mathrm{V}} \in[1 ; 100]$.

\subsection{Model of aggregated and isolated particles}

In order to model metal particles as observed in Fischer-Tropsch cobalt-based catalyst (Humbert et al., 2018), a combination of union and intersection of Boolean model of sphere can be build. Let $A, B$ and $C$ be three Boolean models of spheres with radius following three different log-normal distributions. Let $D$ be the model defined by:

$$
D=(A \cap B) \cup\left(A^{\mathrm{c}} \cap C\right)
$$

Model $A$ represents aggregates, $B$ the particles in the aggregates and $C$ the particles outside the aggregates. Such model still have an analytical covariance (see supplementary materials). Figure 9 shows scattered intensities for four examples of such models with various volume fraction of the $A, B$ and $C$ models.

\subsection{Cox model}

Figure 10 shows two-dimensional cross sections of two realizations of an intersection of two Boolean models of spheres and the corresponding Cox model. Parameters of the models are reported in Table 2. Cox model is generated such as the whole sphere of a 
particle is included in the sphere of an aggregate. The parameters of the Cox model have been adjusted to have an equivalence between the models (same particle radius $R^{(2)}$, same aggregate radius $R^{(1)}$, same volume fraction of particles in aggregates $\left.p^{(2)}\right)$. The volume fraction of aggregates of the Cox model $p^{(1)}$ has been adjusted to obtain the same mean total volume fraction as for the intersection of Boolean models, namely $p=0.21$. As the volume fraction of the Cox model is not analytical, $p^{(1)}$ was adjusted by a dichotomy procedure using several tries comporting 1000 realizations each. The value $p^{(1)}=0.397878$ leads to a mean volume fraction $p=0.2106 \pm 0.00148(95 \%$ confidence interval), compatible with the $p=0.21$ targeted value. For the intersection model, primary spheres are cut at the border of the aggregates whereas they are conserved in the corresponding Cox model. Figure 11 shows the scattered intensities of both models. A subtle difference is observed between the two models in the space scale close to the small spheres radius $\left(q R^{(1)} \approx 6\right)$. We recall that the intensities of the Cox model cannot be computed from analytical covariance as its covariance cannot be determined analytically. Therefore, it is only possible to compute it from projection of realizations.

\section{Discussion}

In the Figure 3, the increased overlapping of spheres for the $\mathrm{p}=0.8$ model compared to the $\mathrm{p}=0.2$ model results in more smoothed oscillations of the intensities. At high $q$, the departure from expected values of the intensities computed from projections is explained by discretization errors. The condition $q \gtrsim 1$ corresponds to distance in the $r$ space lower than $2 \pi$ voxels for which binarization effects become significant in the realizations of the models. It limits the range of $q$ that can be probed to $[2 \pi / N ; 1]$ in

voxel $^{-1}$, where $N$ is the number of voxels for the side of the simulation cube. The same effect is present for the calculation from analytical covariance but is much less present IUCr macros version 2.1.11: 2019/01/14 
as the number of points can be made much larger because only a one-dimensional information is needed.

Underestimation of the intensity at low $q$ (Figure 7) for low volume fraction and overestimation for high volume fraction can be explained by the truncation of the Gamma distribution for large radius during the generation of the realization of the model. Indeed, the realization of the Boolean model in a finite size volume cannot accommodate grains with diameter larger that the cube side. It may be a problem for distributions in size that are not bounded. Therefore, the Gamma distribution has been truncated to ten times the mean radius in number $(10 b c=80$ voxels $)$ and this caused the underestimation of intensity at small $q$. Such effect limits clearly the computation of the SAXS intensity to models which grains have a distribution in size that is rapidly decreasing and not too wide.

The oscillations of the intensity computed from projection around the expected value are attributed to finite-size effect of the simulation. These effects are well known for the computation of structure factor and compressibility of gas from molecular dynamics simulation of periodic systems. Salacuse et al. (1996) have discussed the origin of these effects which are explicit (the number of particle can vary in the simulation volume) or implicit (due to the periodic boundary conditions) and they proposed a way to correct them. Brisard \& Levitz (2013) have proposed another methodology to correct these finite size effects that is more universal and accurate. Their method considers granular media formed by individual particles, and the SAXS intensities are obtained semi-analytically whereas calculation from projection is digital for any arbitrary periodic microstructure. Concerning these finite-size effects, Boolean models should behave as the systems of individual particles described by these authors. For a realization of a model, the number of grains in the simulation volume follows a Poisson law which gives inevitably rise to explicit size effect. The periodic boundary conditions

IUCr macros version 2.1.11: 2019/01/14 
of the realizations are similar to set of individual particles and provokes implicit effects. The computation of an averaged intensity from a large number of realizations strongly diminishes these finite-size effect at the expense of numerous generation of realizations, their projection and computation of intensity. An adaptation of the algorithm proposed by Brisard \& Levitz (2013) to two-dimensional Fourier transform (equation 18) should correct the finite-size effect and allow an accurate computation of the SAXS intensity from a single realization. It is worth mentioning that these finite size effects are not compatible with the definition of a Representative Volume Element (RVE) for the SAXS intensity. Even for high $N$, the fluctuation of intensity at a given $q$ does not converge proportionally with the inverse of $N^{3}$, the volume of the simulation cube (Lantuejoul, 1991). This is confirmed by the results shown in Figure 4 where the variance of intensities are found independent of $q$ and $N$. It does raise question if the denomination of finite-size effect is correct. We have observed this same constant variance for the intersection of two Boolean models of spheres and its corresponding Cox model presented in section 4.5. The relative variance of intensity for a given $\mathbf{q}$ is conjectured to be equal to one. The value 0.5 obtained in Figure 4 is explained by the fact that the calculated intensities are an average of two directions (vertical and horizontal).

Numerical costs of the calculation from projection are essentially limited by the simulation of a realization (Table 1). The size of the volume $N$ is limited by the available memory. Size of $N=2048$ mobilizes 8 Go of memory just to store the realization and is the practical limit on a standard computer. Moreover, the number of implanted grains is proportional to $N^{3}$ so that simulation time grows quickly when the size $N$ grows. Finite size effects are not removed even for sizes as large as $N=2048$ and have to be mitigated by averaging large number of realizations. The needed time scales as the number of realizations $n$, such as the variance of the intensity. The 
relative variance of the computed intensities (Figure 4) is constant for the whole $q$ range independently of the size of the simulation. It means that there is no interest to perform realizations on large volumes, provided that the volume is big enough to accommodate the largest sphere. The order of magnitude of this constant variance close to $1 / 2$ allows to predict the standard deviation of the computed intensity to about $\sqrt{n / 2}$, with $n$ the number of realizations. This large variance necessitates a high number $n$ of realizations to obtain relative uncertainties close to experimental ones that can be found lower than the percent.

Contrariwise, the calculation of the SAXS intensity from analytical covariance is free from explicit size effects and implicit ones can be made very small using very large range for sampling the covariance. The memory requirements are low: 8 Mo for a $2^{18}$ simulation domain size. Running times are also very small (less than a second) since only a one-dimensional FFT and a numerical differentiation is needed. The last method, calculation from the numerical evaluation of the covariance, has not been explored. It has the drawbacks of important finite size effects and the need of large memory for performing a three-dimensional FFT. Moreover the numerical differentiation in equation 20 with relatively large $\Delta q$ leads to large numerical inaccuracies.

The computation of the SAXS intensity from analytical covariance seems to be the method of choice. It allows the simulation of true multi-scales models, even up to some showing a fractal behavior over several orders of magnitude of length scale as shown in Figure 8. Such power law dependence has been predicted by Schmidt (1982) for independently randomly oriented objects that have a size distribution with a powerlaw tail. Here, we observe the same behavior for a consolidated media and a size distribution with a tail decreasing faster than a power law. The model of aggregated and isolated particles of section 4.4 is able to qualitatively reproduce the experimental ASAXS intensity of cobalt-baser Fischer-Tropsch Catalyst (Humbert et al., 2018).

IUCr macros version 2.1.11: 2019/01/14 
However, it has the strong drawback that only Boolean models with an analytical covariance may be used to interpret SAXS intensity. This strongly limits the geometry of the grains to spheres or cylinders (Willot, 2017) and to union and/or intersection of Boolean models. For example, microstructures as Cox model of aggregates of platelets for modeling alumina supports or Cox model of spherical aggregates of spherical particles for modeling Fischer-Tropsh catalysts are out of reach with this approach. For one-scale Boolean models of grains for which analytical covariance is not known, a mixed approach could be settled. The covariogram may be numerically evaluated (Moreaud et al., 2012), the covariance obtained by equation 5 and the SAXS intensity computed from the covariance. Nevertheless, the difference between an intersection of Boolean model of spheres and the corresponding Cox model is very subtle as shown Figure 11. As morphological models have parameters with a geometrical meaning, it can lead to a two steps process to interpret SAXS intensity by morphological models. First, the parameters of a Boolean model with known covariance could be fit by least squares to experimental data. These parameters could then be refined with the corresponding Cox model, limiting an expensive optimum search arising from computation by projection. The calculation from projection, whereas requiring much more memory and computation time, is more universal as any binarized microstructure may be processed. It will strongly benefit of the implementation of a correction for finite size effects. Moreover, this approach has the advantage of needing only the projection of the microstructure. Using the infinite divisibility of Boolean models and extending the work of Jeulin \& Moreaud (2011) to projection calculation, it may be possible to overcome the memory limitations to be able to compute larger simulation volumes to handle multi-scale microstructures.

IUCr macros version 2.1.11: 2019/01/14 


\section{Conclusion}

The use of Boolean model is important for the interpretation of SAXS intensity of system encountered in heterogeneous catalysis. The three common methods that are found in literature to compute the SAXS intensity from a Boolean model have been exposed. Calculation from known analytical covariance of union and intersection of Boolean models allows to model complex microstructures including spherical particles with exponential, Gamma or log-normal distribution in radius. Such approach can model fractal-like behavior and systems of aggregating particles. Comparison of an intersection of Boolean model of spheres and the corresponding Cox model is very subtle. This open interesting perspectives to interpret SAXS intensities of system of isotropic particles and aggregates with such union and intersection of Boolean models with analytical covariance. Calculation of intensities from projection can be applied on any periodic digital representation of a microstructure. However, it appears it is prone to strong finite size effects. These effects may be mitigated by computing the average of numerous realizations of the model at the expense of long computing times. With a proper implementation of a correction for finite size effects, this method is however more attractive as not restricted to grains of simple geometry and to union or intersection of Boolean models.

Authors warmly thank the two anonymous reviewers and Editor of the journal for their advice to improve the early version of the manuscript.

\section{References}

Beaucage, G. (1995). Journal of Applied Crystallography, 28(6), 717-728.

Bressler, I., Pauw, B. \& Thünemann, A. (2015). Journal of Applied Crystallography, 48(3), 962-969.

Brisard, S., Chae, R. S., Bihannic, I., Michot, L., Guttmann, P., Thieme, J., Schneider, G., Monteiro, P. J. \& Levitz, P. (2012). American Mineralogist, 97(2-3), 480-483.

Brisard, S. \& Levitz, P. (2013). Physical Review E, 87(1), 013305.

Datye, A. K., Xu, Q., Kharas, K. C. \& McCarty, J. M. (2006). Catalysis today, 111(1-2), $59-67$.

Dingerdissen, U., Martin, A., Herein, D. \& Wernicke, H. J. (2008). In Handbook of Heterogeneous Catalysis, pp. 37-56. Wiley Online Library.

IUCr macros version 2.1.11: 2019/01/14 
Friedrich, H., de Jongh, P. E., Verkleij, A. J. \& de Jong, K. P. (2009). Chemical reviews, 109(5), 1613-1629.

Gille, W. (2011). Computers \& Structures, 89(23-24), 2309-2315.

Gommes, C. J. (2018). Microporous and Mesoporous Materials, 257, 62-78.

Gommes, C. J., Jiao, Y. \& Torquato, S. (2012). Physical Review E, 85(5), 051140.

Granqvist, C. \& Buhrman, R. (1976). Journal of Catalysis, 42(3), 477-479.

Humbert, S., Desjouis, G., Bizien, T., Lemaitre, L., Taleb, A.-L., Dalverny, C., Sorbier, L. \& Gay, A.-S. (2018). Journal of Catalysis, 366, 202-212.

Jeulin, D. (1996). In European Workshop on Application of Statistics and Probabilities in Wood Mechanics, Bordeaux, pp. 22-23.

Jeulin, D. (2012). Comptes Rendus Mécanique, 340(4-5), 219-229.

Jeulin, D. \& Moreaud, M. (2011). Image Analysis \& Stereology, 26(3), 121-127.

Kak, A. \& Slaney, M. (1988). Principles of Computerized Tomographic Imaging, vol. 33, chap. 3, pp. 56-57. Philadelphia: SIAM.

Koch, K., Ohser, J. \& Schladitz, K. (2003). Advances in Applied Probability, 35(3), 603-613.

Konarev, P., Volkov, V., Sokolova, A., Koch, M. \& Svergun, D. (2003). Journal of Applied Crystallography, 36(5), 1277-1282.

Lantuejoul, C. (1991). Journal of Microscopy, 161(3), 387-403.

Levitz, P. \& Tchoubar, D. (1992). Journal de Physique I, 2(6), 771-790.

Li, T., Senesi, A. J. \& Lee, B. (2016). Chemical reviews, 116(18), 11128-11180.

Lomić, G. A., Kiš, E. E., Bošković, G. C. \& Marinković-Nedučin, R. P. (2004). Acta Periodica Technologica, (35), 67-77.

Matheron, G. (1967). Eléments pour une théorie des milieux poreux. Paris: Masson.

Matheron, G. (1975). Random Sets and Integral Geometry. New York: Wiley.

Moreaud, M. (2006). Propriétés morphologiques multi-échelles et prévision du comportement diélectrique de nanocomposites. Ph.D. thesis, Ecoles des Mines de Paris. URL: https://pastel.archives-ouvertes.fr/pastel-00002221

Moreaud, M., (2018). plug im! an open access and customizable software for signal and image processing. URL: https://www.plugim.fr

Moreaud, M., Jeulin, D., Morard, V. \& Revel, R. (2012). Journal of Microscopy, 245(2), 186-199.

Munnik, P., De Jongh, P. E. \& De Jong, K. P. (2014). Journal of the American Chemical Society, 136(20), 7333-7340.

Salacuse, J., Denton, A. \& Egelstaff, P. (1996). Physical Review E, 53(3), 2382-2389.

Schmidt, P. (1982). Journal of Applied Crystallography, 15(5), 567-569.

Schmidt-Rohr, K. (2007). Journal of Applied Crystallography, 40(1), 16-25.

Sonntag, U., Stoyan, D. \& Hermann, H. (1981). Physica Status Solidi (a), 68(1), 281-288.

Thomas, J. \& Terasaki, O. (2002). Topics in catalysis, 21(4), 155-159.

Wang, H., Pietrasanta, A., Jeulin, D., Willot, F., Faessel, M., Sorbier, L. \& Moreaud, M. (2015). Journal of microscopy, 260(3), 287-301.

Weckhuysen, B. M. (2009). Angewandte Chemie International Edition, 48(27), 4910-4943.

Willot, F. (2017). Journal of Contemporary Mathematical Analysis, 52(6), 305-315.

Witte, P. T., Boland, S., Kirby, F., van Maanen, R., Bleeker, B. F., de Winter, D. M., Post, J. A., Geus, J. W. \& Berben, P. H. (2013). ChemCatChem, 5(2), 582-587.

IUCr macros version 2.1.11: 2019/01/14 
Table 1. Typical number of implanted grains and running times for one realization of a $512^{3}$ volume of a Boolean model of spheres of 20 voxels radius.

\begin{tabular}{lcc}
\hline$p$ & 0.2 & 0.8 \\
\hline Number of grains & 900 & 6400 \\
Running time (s) & 15 & 45 \\
\hline
\end{tabular}

Table 2. Parameters for two-scales models of spheres of Figure 11.

\begin{tabular}{lcc}
\hline & Intersection & Cox model \\
\hline$p^{(1)}$ & 0.3 & 0.397878 \\
$R^{(1)}$ & 64 & 64 \\
$p^{(2)}$ & 0.7 & 0.7 \\
$R^{(2)}$ & 8 & 8 \\
\hline
\end{tabular}

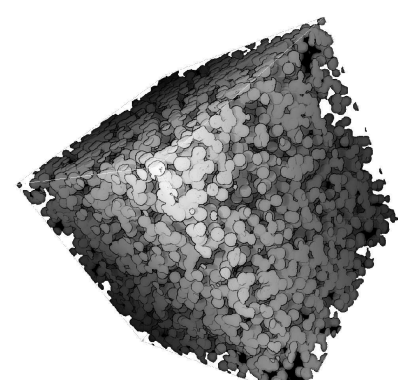

(a)

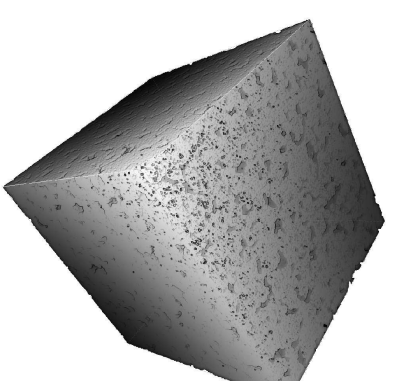

(b)

Fig. 1. Surface rendering of a realization of a one-scale Boolean model of spheres with constant radius $R=20$ voxels with (a) $p=0.2$ and (b) $p=0.8$. 


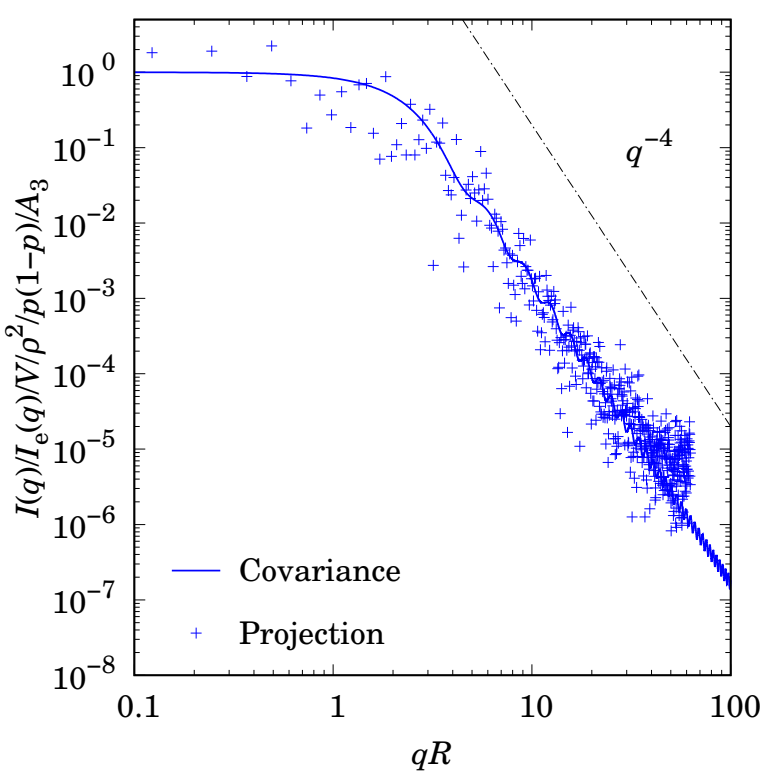

Fig. 2. Comparison of calculated SAXS intensity from analytical covariance (lines) and projection (symbols) for one realization (1024 ${ }^{3}$ voxels volume) of a one-scale Boolean model of spheres with constant radius $R=20$ and volume fraction $p=0.8$.

IUCr macros version 2.1.11: 2019/01/14 


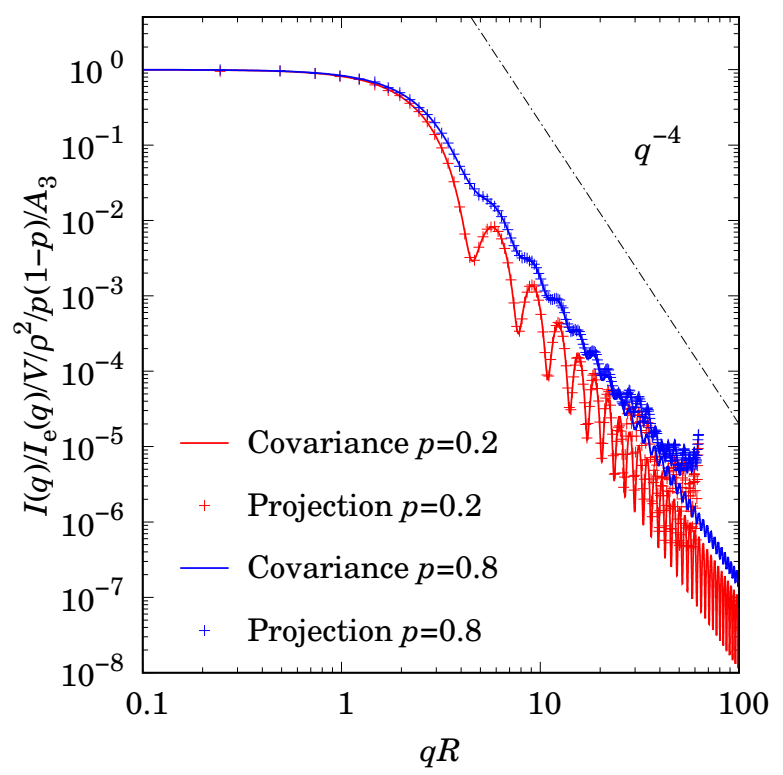

(a)

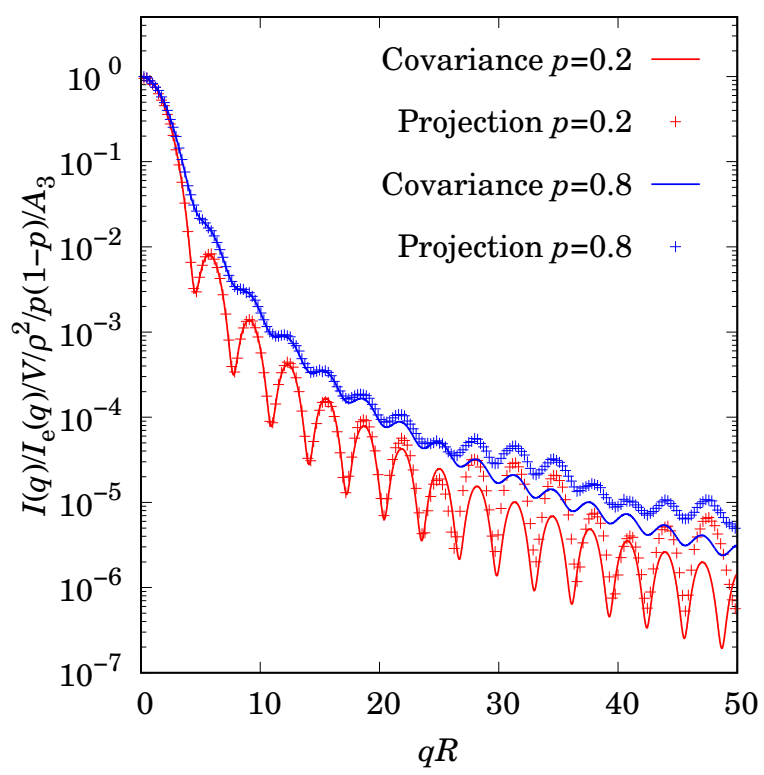

(b)

Fig. 3. Comparison of calculated SAXS intensity from analytical covariance (lines) and projection (symbols) for 1000 realizations ( $512^{3}$ voxels volume) of a one-scale Boolean model of spheres with constant radius $R=20$. (a) log-log plot (b) semi-log plot. 


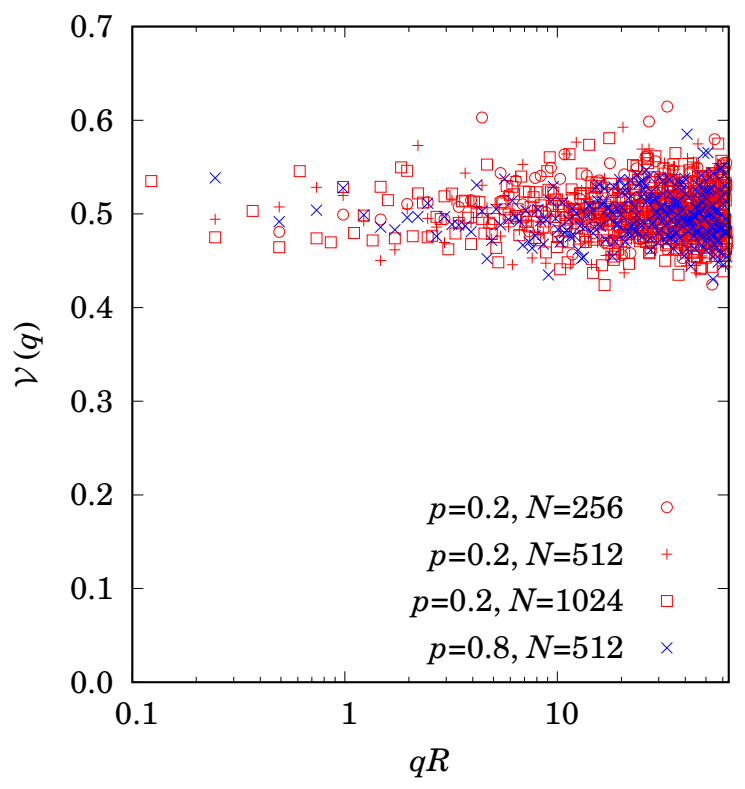

Fig. 4. Relative variance of $I_{\text {proj }}(q)$, the scattered intensity computed from projection, for Boolean models of spheres with constant radius $R=20$ evaluated from 1000 realizations.

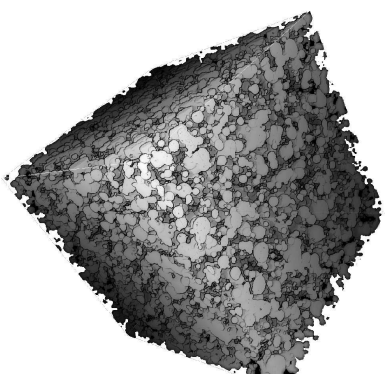

(a)

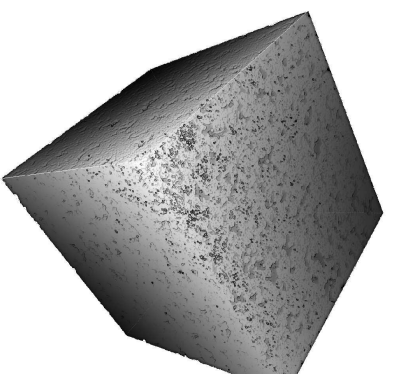

(b)

Fig. 5. Surface rendering of a realization of a one-scale Boolean model of spheres with radius following a Gamma distribution of scale parameter $b=4$ and shape parameter $c=2$ with (a) $p=0.2$ and (b) $p=0.8$. 


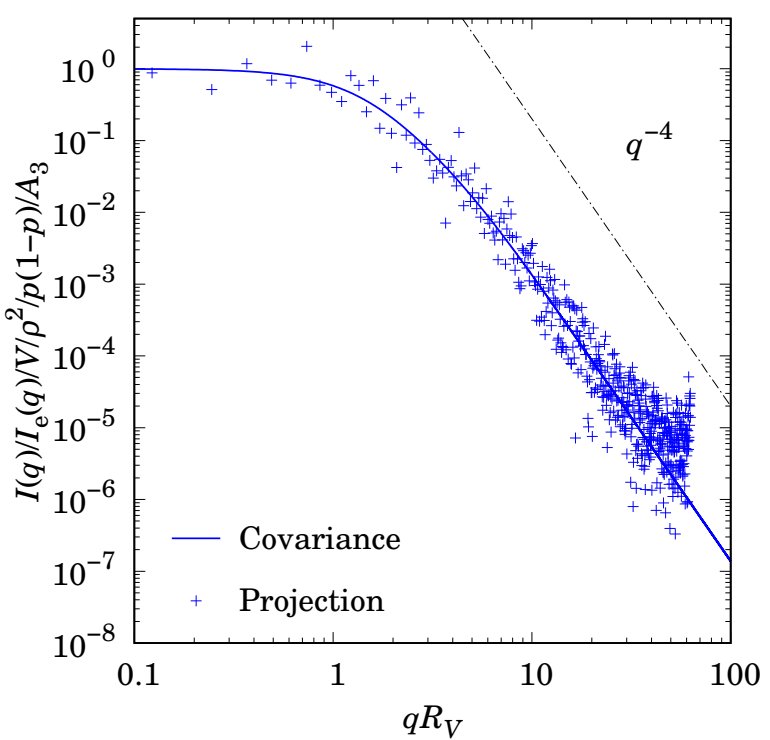

Fig. 6. Comparison of calculated SAXS intensity from analytical covariance (lines) and projection (symbols) for one realization (1024 ${ }^{3}$ voxels volume) of a one-scale Boolean model of spheres with radius $R$ following a Gamma distribution of scale parameter $b=4$ and shape parameter $c=2$ and a volume fraction $p=0.8$. 


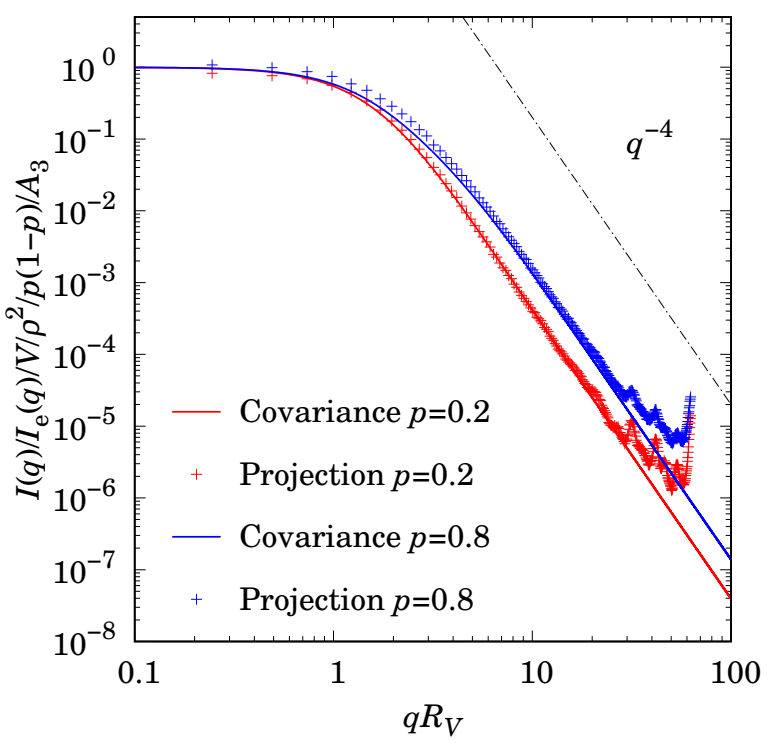

(a)

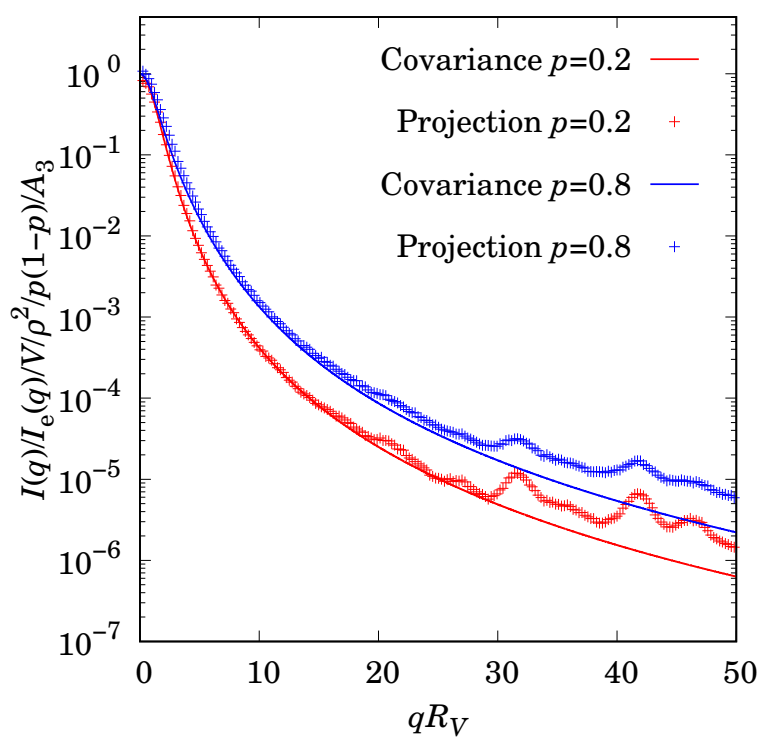

(b)

Fig. 7. Comparison of calculated SAXS intensity from analytical covariance (lines) and projection (symbols) for 1000 realizations ( $512^{3}$ voxels volume) of a one-scale Boolean model of spheres with radius $R$ following a Gamma distribution of scale parameter $b=4$ and shape parameter $c=2$. 


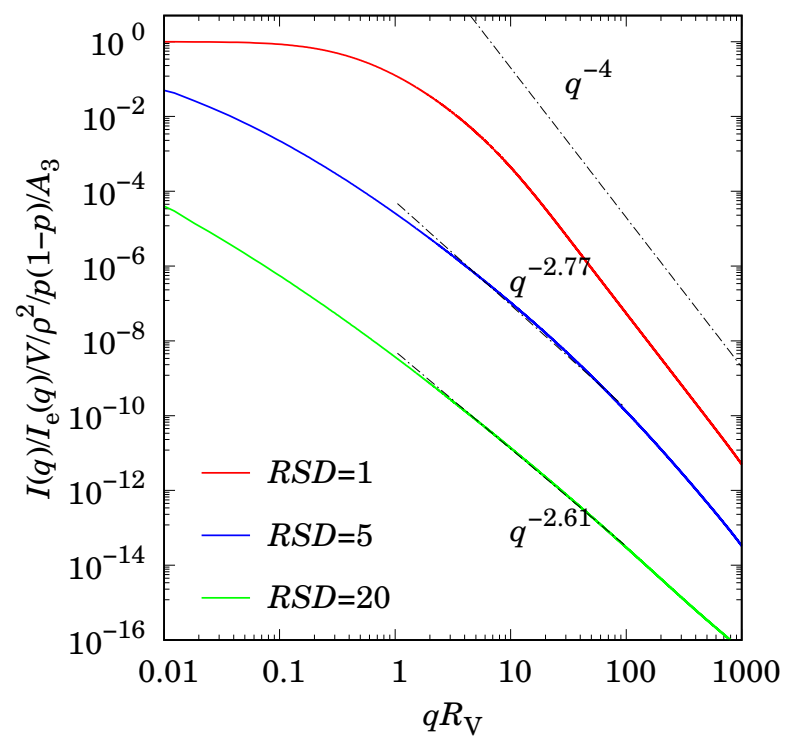

Fig. 8. SAXS intensity from analytical covariance for Boolean model of spheres with radius following log-normal distributions and a volume fraction $p=0.2$. Dashed lines are power law least square fit of the intensities for $q R_{\mathrm{V}} \in[1 ; 100]$.

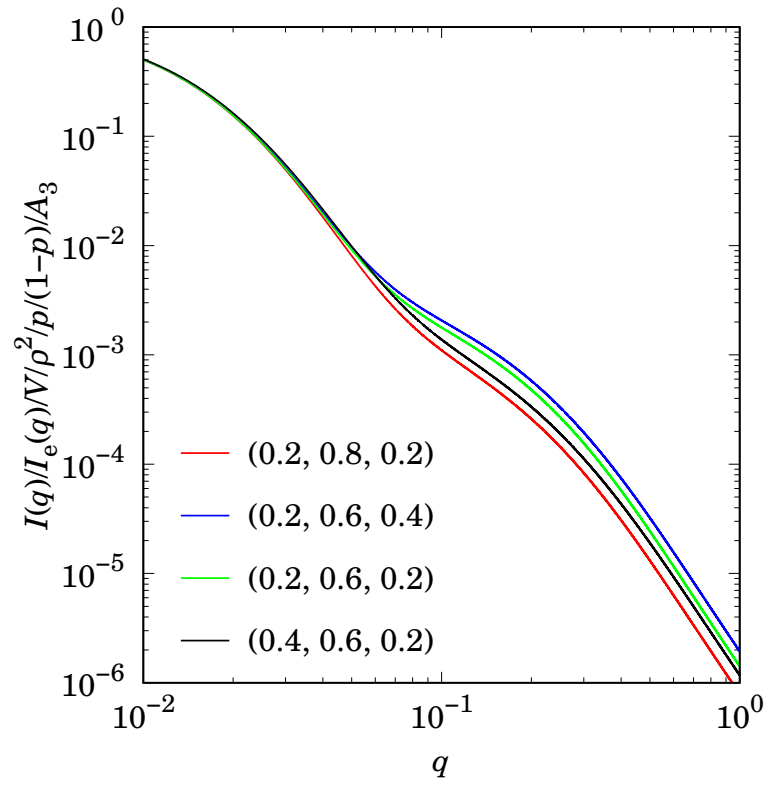

Fig. 9. SAXS intensity from analytical covariance for model of aggregated and isolated particles (equation 23). Numbers in legend report the volumic fraction of the primary models $\left(p^{(A)}, p^{(B)}, p^{(C)}\right) \cdot R_{\mathrm{V}}^{(A)}=100, R_{\mathrm{V}}^{(B)}=R_{\mathrm{V}}^{(C)}=10$.

IUCr macros version 2.1.11: 2019/01/14 


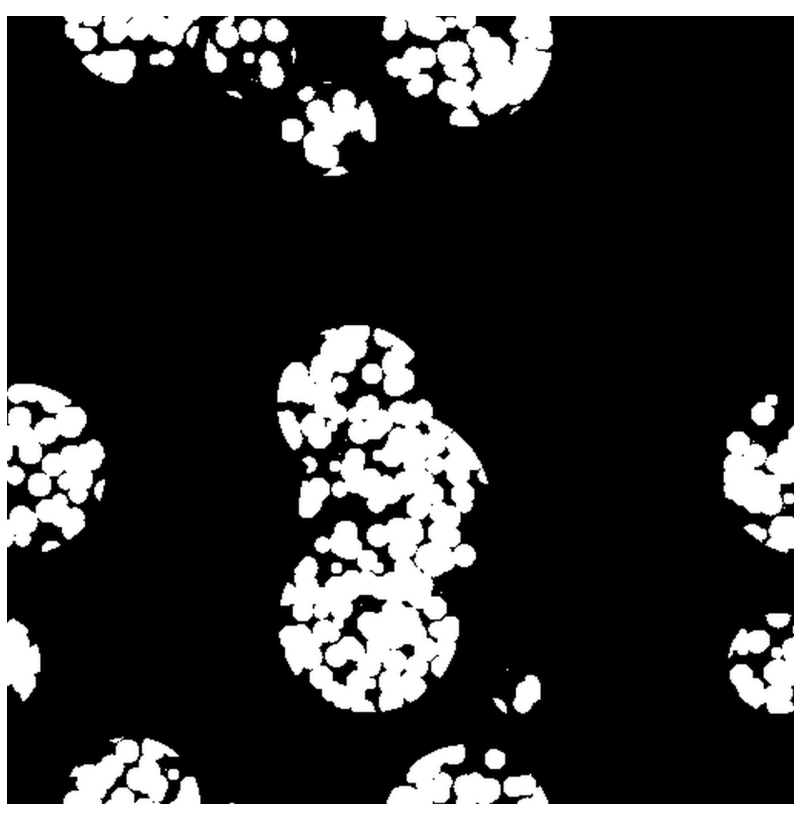

(a)

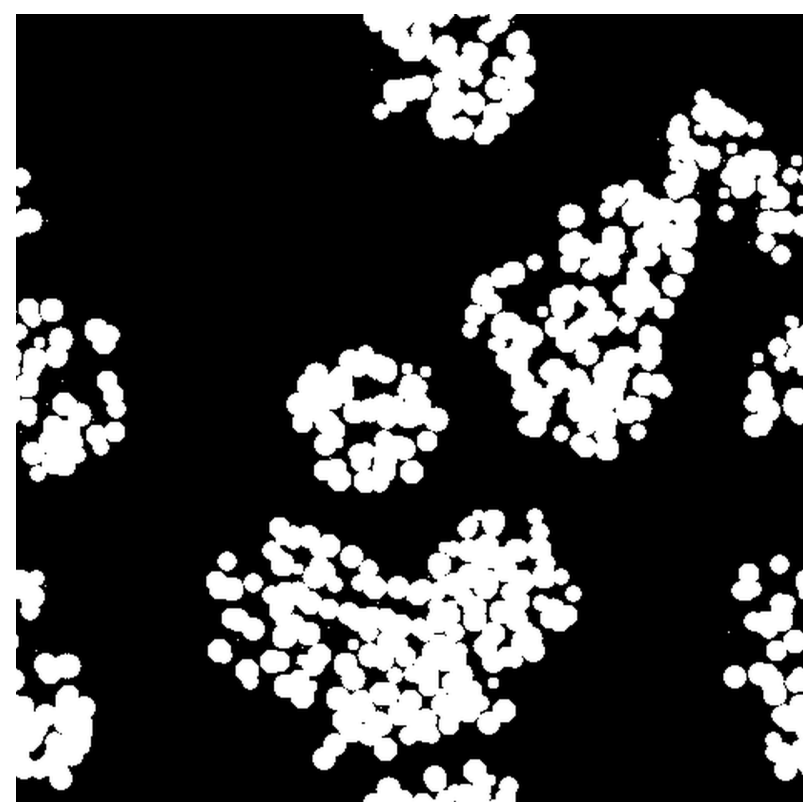

(b)

Fig. 10. Two dimensional cross sections of realizations of (a) an intersection of two Boolean models of spheres (b) the corresponding Cox model. Parameters of the models are reported in Table 2 . 


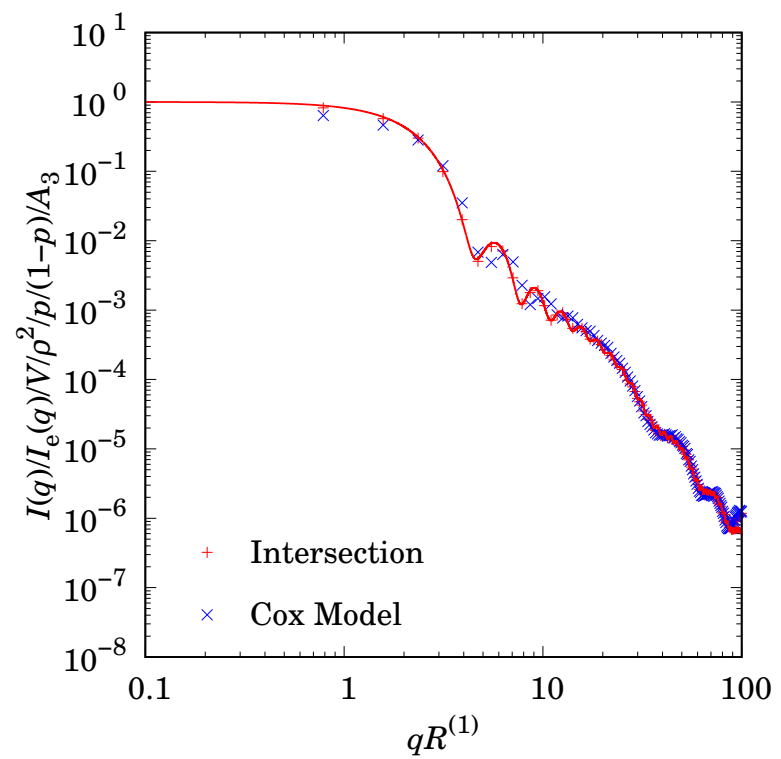

Fig. 11. SAXS intensity for an intersection of two Boolean models of spheres with constant radius (red crosses from projection, line from covariance) and the corresponding two-scales Cox model (blue crosses from projection). Average from 1000 realizations ( $512^{3}$ voxels volume). Parameters of the models are reported in Table 2 .

\section{Synopsis}

Methods for computing SAXS intensity for Boolean models of spheres from known covariance or from projection of realizations are reviewed and compared. 